\title{
The ratio of cytotoxic lymphocytes to M2-like macrophages is prognostic in immunogenic tumors
}

Authors: Artur Mezheyeuski*, Max Backman¹, Johanna Mattsson¹, Alfonso Martín-Bernabé2, Chatarina Larsson ${ }^{1}$, Ina Hrynchyk ${ }^{3}$, Klara Hammarström ${ }^{1}$, Simon Ström ${ }^{1}$, Joakim Ekström ${ }^{1}$, Siarhei Mauchanski ${ }^{4}$, Salome Khelashvili ${ }^{4}$, Margrét Agnarsdóttir ${ }^{5}$, Per-Henrik Edqvist ${ }^{1}$, Jutta Huvila $^{6}$, Ulrika Segersten ${ }^{7}$, Per-Uno Malmström ${ }^{7}$, Johan Botling ${ }^{1}$, Björn Nodin ${ }^{8}$, Charlotta Hedner $^{8}$, David Borg ${ }^{8}$, Jenny Brändstedt ${ }^{8}$, Hanna Sartor ${ }^{9}$, Karin Leandersson ${ }^{10}$, Bengt Glimelius ${ }^{1}$, Anna Portyanko ${ }^{4}$, Fredrik Ponten ${ }^{1}$, Karin Jirström ${ }^{8}$, Patrick Micke ${ }^{1} \dagger$ and Tobias Sjöblom ${ }^{1} \uparrow$.

\section{Affiliations:}

${ }^{1}$ Dept. of Immunology, Genetics and Pathology, Uppsala University, Rudbeck Laboratory, 751 85 Uppsala, Sweden.

${ }^{2}$ Dept. of Oncology-Pathology, Cancer Center Karolinska, Karolinska Institutet, Karolinska vägen, A2:07, 17164 Solna, Sweden.

${ }^{3}$ City Clinical Pathologoanatomic Bureau, Minsk 220116, Republic of Belarus.

${ }^{4}$ N.N. Alexandrov National Cancer Centre of Belarus, Lesnoy, Minsk, 223040, Republic of Belarus.

${ }^{5}$ Dept. of Immunology, Genetics and Pathology, Clinical and experimental pathology, Uppsala University, Rudbeck Laboratory, 75185 Uppsala, Sweden.

${ }^{6}$ Dept. of Pathology, University of Turku, 20500 Åbo, Finland.

${ }^{7}$ Dept. of Surgical Sciences, Uppsala University, Akademiska sjukhuset, 75185 Uppsala, Sweden.

${ }^{8}$ Dept. of Clinical Sciences Lund, Division of Oncology and Therapeutic Pathology, Lund University, Hämtställe 66, BMC F12, 22184 Lund, Sweden.

${ }^{9}$ Diagnostic Radiology, Dept. of Translational Medicine, Lund University, Skåne University Hospital, Carl-Bertil Laurells gata 9, 20502 Malmö, Sweden.

${ }^{10}$ Cancer Immunology, Dept. of Translational Medicine, Lund University, J Waldenströms gata 35, 20502 Malmö, Sweden.

*Correspondence to: artur.mezheyeuski@igp.uu.se.

$\dagger$ equal contribution

One Sentence Summary: The CD8/M2 ratio in tumor tissue defines prognosis in immunogenic cancers 


\begin{abstract}
Immune cells in the microenvironment shape tumor development and progression. The prognostic value of T-cell-based immune scores exceeds those of clinical parameters in colon cancer, but reflects only a part of the anti-tumor immune response. Here, we assessed 15 distinct immune cell classes and identified a simple prognostic signature based on pro- and anti-tumoral immune cells in the tumor microenvironment. The ratio of cytotoxic lymphocytes to tumor supportive macrophages predicted survival better than the state-of-art immune score in colon cancer and had the highest relative contribution to survival prediction when compared to established clinical parameters. This signature was prognostic also in other cancers with high mutation burden, such as those of the lung, bladder, esophagus, and melanomas, supporting broad clinical applicability.
\end{abstract}




\section{Main text}

Colorectal cancer (CRC) is the fourth most common and the second most lethal type of cancer (1). The traditional TNM classification, cancer gene mutations and expression profiles identify more homogeneous subgroups among the intrinsically heterogeneous CRC tumors (2). Recently, the immune response was acknowledged as a prognostic factor in CRC (3). An immune scoring system that evaluates the abundance of $\mathrm{CD}^{+}$and $\mathrm{CD} 8^{+} \mathrm{T}$ cells in resected tumors was recently validated as an independent prognostic factor in colon cancer stage I-III, surpassing established clinical parameters such as $\mathrm{T}$ and $\mathrm{N}$ stage $(4,5)$. The Immunoscore ${ }^{\circledR}$, although of proven validity, quantitates only a limited subset of anti-tumoral immune cells, but a growing body of evidence supports association of additional immune cell types, including B cells and NK cells, with better outcome (6-9). In addition, T-regulatory lymphocytes and M2-polarised macrophages residing in the tumor microenvironment have been connected to tumor progression (10), suggesting protumoral effects and prognostic potential of these immune suppressive cells. The aims of this study were (1) to generate a comprehensive overview of the immune landscape in CRC by in situ analysis of 15 distinct subclasses of T- and B-lymphocytes, myeloid cells and NK cells, (2) to identify the immune cell signature with the highest prognostic value, and (3) to assess the prognostic ability of this signature in other tumor types. 
To quantitate the different immune cell subsets, we performed immunohistochemistry (IHC) and multispectral imaging enabling multiplex labeling of markers in tumor tissue. We used two IHC panels, each consisting of antibodies to five immune markers, for visualization of adaptive and innate immune cells. After cell segmentation of digitized tissue sections, the co-expression patterns of these markers allowed for immune cell classification into distinct subgroups $(\operatorname{see}(9,11,12)$ and Supplementary Materials and Methods) (Fig 1A). The major immune cell lineages were defined by single markers (CD4, CD8, CD45RO, CD68 and CD163). By marker co-expression we identified memory CD4 and CD8 lymphocytes, classical CD4 ${ }^{+}$T-regulatory and CD8 ${ }^{+}$Treg cells. For natural killer (NK) cells, we required co-expression of two markers (CD56 and NKp46) to classify a cell as NK, along with CD3 expression to classify as NK T (NKT) cell. Finally, the monocyte/macrophage lineage was sub-divided into M1-like macrophages (CD68 $\left.{ }^{+}{ }^{-163^{-}}\right)$, M2like macrophages $\left(\mathrm{CD} 68^{+} \mathrm{CD} 163^{+}\right)$and $\mathrm{CD} 68^{-} \mathrm{CD} 163^{+}$cells.

First, we evaluated the prognostic impact of the densities of the different immune cells in therapy naïve stage I-III colon cancers $(n=286)$. Two cell classes demonstrated association of cell density with overall survival (OS), namely $\mathrm{CD}^{+} \mathrm{T}$ lymphocytes (positive association, $p=0.042$ ) and M2like macrophages (negative association, $p=0.004$ ) (Fig 1B). Neither the pan-macrophage marker CD68 alone nor CD163, which is considered a marker of M2 differentiation, alone were associated with survival, whereas a more stringently defined M2-like macrophage subset expressing both CD68 and CD163 was (Fig 1B and C). Across all tumors, the $\mathrm{CD}^{+} 8^{+} \mathrm{CD} 163^{+} \mathrm{M} 2$-like macrophages constituted only $5 \%$ of the $\mathrm{CD}^{+} 8^{+}$macrophages and $23 \%$ of the $\mathrm{CD} 163^{+}$cells (Fig 1D), but demonstrated substantial inter-patient heterogeneity with cell density ranging from 0 to 1080 cells $/ \mathrm{mm}^{2}$ of tumor tissue (Fig 1E). Hypothesizing that these two immune cell types capture the 
interplay between anti- and pro-tumoral aspects of the immune microenvironment, we created a signature of immune activation (SIA) based on the relative infiltration levels of $\mathrm{CD}^{+}$cells and M2-like macrophages (Fig 1F and Supplementary materials).

To determine the prognostic value of SIA with regard to OS and recurrence-free survival (RFS), we transformed into a three-level categorized variable, using an unbiased approach with 33.3 and 66.6 percentiles as cutoffs. For comparison, we generated an Immunoscore-like metric (IS) by quantifying densities of $\mathrm{CD}^{+}$and $\mathrm{CD}^{+}$cells at the tumor center and invasive margin (4). Both IS and SIA demonstrated strong associations with OS and RFS in colon cancer stage I-III (Fig 2A and B). Interestingly, in a multivariate Cox model adjusted for $\mathrm{pT}$ stage, $\mathrm{pN}$ stage, patient age, gender and MSI status, both SIA and IS were independent predictors for OS and RFS (Table 1). Next, we compared the predictive ability of SIA to IS and well-known clinical risk factors. Integrative time-dependent estimation of the area under receiver-operator curve (iAUC) identified T stage as the strongest clinical predictor for OS (median iAUC 0.58) and N stage for RFS (median iAUC 0.58) (Fig 2C). However, both clinical risk factors and IS were inferior to SIA (median iAUC 0.59 for OS and RFS). Adding SIA to the model with combined clinical parameters improved the predictive ability (median iAUC 0.66 and 0.67 for OS and RFS). Finally, integration of clinical parameters, IS and SIA in one model resulted in median iAUC 0.68 and 0.69 for OS and RFS, respectively. The relative contribution to OS prediction was higher for SIA than for T and $\mathrm{N}$ stage, and when including IS in the model, the relative contribution of SIA and IS exceeded $50 \%$ and clearly surpassed the known clinical factors (Fig 2D). Because of the clinical need to identify high-risk tumors in stage II colon cancer patients, we analyzed this patient subgroup separately $(n=117)$ and observed similar results with SIA stratifying high and low-risk disease (Fig 
S1A). Next, we assessed CRC patients with metastatic disease $(n=66)$, stratified into three equallysized terciles according to SIA and observed longer OS in the SIA-high group (Fig S1B). Thus, SIA demonstrated independent prognostic performance superior to the strongest clinical predictors ( $\mathrm{T}$ and $\mathrm{N}$ stage), added substantial value to the multivariate prediction model in colon cancer patients of stages I-III, and demonstrated prognostic ability in stage II colon cancer and in metastatic CRC patients.

Finally, we asked whether SIA was prognostic also in other cancers. We hypothesized that SIA would likely be of highest utility in tumor types with strong immunogenic properties (13). We ranked tumor types according to the number of mutations and neoantigens (Fig 3A), and analyzed four cohorts of tumors characterized by high counts, namely melanoma (14), lung carcinoma (15), bladder urothelial cancer $(16,17)$ and gastroesophageal adenocarcinomas $(18-20)$. We also included two tumor types with low mutation and neoantigen density, endometrial $(21,22)$ and ovarian cancer $(23,24)$. Patients were stratified in terciles according to SIA, except for melanoma where the median was used since $41 \%$ of patients had the highest possible SIA value. High SIA was associated with longer survival in the four tumor types with high mutation and neoantigen count $(p=0.001-0.037)$ while no association was seen in endometrial $(p=0.996)$ and ovarian $(p=0.399)$ cancers (Fig 3B). Further, SIA surpassed IS for prediction of OS in the four cohorts, demonstrating median iAUC ranging from 0.55 in bladder cancer to 0.61 in melanoma (Fig 3C). Thus, the SIA is a prognostic factor in at least five tumor types.

In summary, by immune cell sub-classification we confirmed the prognostic impact of CD $8^{+}$cell infiltration and revealed a prognostic subset of macrophages that was undetectable using a single- 
marker approach. The relationship of $\mathrm{CD} 68^{+} \mathrm{CD} 163^{+}$macrophages polarized towards the $\mathrm{M} 2$ phenotype to anti-tumoral $\mathrm{CD}^{+}$cells provided a prognostic metric for the balance between proand anti-tumoral immunity. As both SIA and the Immunoscore-like score were independent variables in the multivariate analysis, these two metrics presumably reflect different aspects of tumor immunity. The SIA, unlike Immunoscore, does not require independent assessment of the tumor central region and invasive margin, and is prognostic in at least five tumor types. Given the $\sim 6.7 \times 10^{6}$ new cases and $>4.3 \times 10^{6}$ cancer-related deaths annually in these five cancers $(25)$, the SIA has potential to enhance the reliability of prognosis prediction, improve the identification of high-risk patients and improve therapy decisions for $>4 \times 10^{6}$ patients per year, thus motivating the introduction of a TNM-Immune cancer classification (26). 


\section{REFERENCES}

1. F. Bray et al., Global cancer statistics 2018: GLOBOCAN estimates of incidence and mortality worldwide for 36 cancers in 185 countries. CA Cancer J Clin 68, 394-424 (2018).

2. J. Guinney et al., The consensus molecular subtypes of colorectal cancer. Nat Med 21, 1350-1356 (2015).

3. J. I. Quezada-Marin et al., Gastrointestinal tissue-based molecular biomarkers: a practical categorisation based on the 2019 World Health Organization classification of epithelial digestive tumours. Histopathology 77, 340-350 (2020).

4. F. Pages et al., International validation of the consensus Immunoscore for the classification of colon cancer: a prognostic and accuracy study. Lancet 391, 2128-2139 (2018).

5. J. Galon et al., Type, density, and location of immune cells within human colorectal tumors predict clinical outcome. Science 313, 1960-1964 (2006).

6. S. Edin et al., The Prognostic Importance of CD20(+) B lymphocytes in Colorectal Cancer and the Relation to Other Immune Cell subsets. Sci Rep 9, 19997 (2019).

7. A. Coppola et al., NK Cell Inflammation in the Clinical Outcome of Colorectal Carcinoma. Front Med (Lausanne) 2, 33 (2015).

8. J. Berntsson, B. Nodin, J. Eberhard, P. Micke, K. Jirstrom, Prognostic impact of tumour-infiltrating B cells and plasma cells in colorectal cancer. Int J Cancer 139, 1129-1139 (2016).

9. S. Lundgren et al., Topographical Distribution and Spatial Interactions of Innate and Semi-Innate Immune Cells in Pancreatic and Other Periampullary Adenocarcinoma. Front Immunol 11, 558169 (2020).

10. R. D. Schreiber, L. J. Old, M. J. Smyth, Cancer immunoediting: integrating immunity's roles in cancer suppression and promotion. Science 331, 1565-1570 (2011).

11. A. Mezheyeuski et al., Multispectral imaging for quantitative and compartment-specific immune infiltrates reveals distinct immune profiles that classify lung cancer patients. The Journal of pathology 244, 421-431 (2018).

12. S. Lundgren et al., Quantitative, qualitative and spatial analysis of lymphocyte infiltration in periampullary and pancreatic adenocarcinoma. Int J Cancer 146, 3461-3473 (2020).

13. T. N. Schumacher, R. D. Schreiber, Neoantigens in cancer immunotherapy. Science 348, 69-74 (2015).

14. S. Stromberg et al., Selective expression of Syntaxin-7 protein in benign melanocytes and malignant melanoma. $J$ Proteome Res 8, 1639-1646 (2009).

15. P. Micke et al., The Impact of the Fourth Edition of the WHO Classification of Lung Tumours on Histological Classification of Resected Pulmonary NSCCs. J Thorac Oncol 11, 862-872 (2016).

16. T. Hemdan et al., The prognostic value and therapeutic target role of stathmin-1 in urinary bladder cancer. $B r J$ Cancer 111, 1180-1187 (2014).

17. A. Mezheyeuski et al., Fibroblasts in urothelial bladder cancer define stroma phenotypes that are associated with clinical outcome. Sci Rep 10, 281 (2020).

18. M. C. Svensson et al., The integrative clinical impact of tumor-infiltrating T lymphocytes and NK cells in relation to B lymphocyte and plasma cell density in esophageal and gastric adenocarcinoma. Oncotarget 8, 72108-72126 (2017).

19. M. C. Svensson et al., Expression of PD-L1 and PD-1 in Chemoradiotherapy-Naive Esophageal and Gastric Adenocarcinoma: Relationship With Mismatch Repair Status and Survival. Front Oncol 9, 136 (2019).

20. M. Jeremiasen et al., Tumor-Associated CD68(+), CD163(+), and MARCO(+) Macrophages as Prognostic Biomarkers in Patients With Treatment-Naive Gastroesophageal Adenocarcinoma. Front Oncol 10, 534761 (2020).

21. P. H. Edqvist et al., Loss of ASRGL1 expression is an independent biomarker for disease-specific survival in endometrioid endometrial carcinoma. Gynecol Oncol 137, 529-537 (2015).

22. J. Huvila et al., Combined ASRGL1 and p53 immunohistochemistry as an independent predictor of survival in endometrioid endometrial carcinoma. Gynecol Oncol 149, 173-180 (2018).

23. G. Berglund, S. Elmstahl, L. Janzon, S. A. Larsson, The Malmo Diet and Cancer Study. Design and feasibility. J Intern Med 233, 45-51 (1993).

24. B. Nodin et al., Increased androgen receptor expression in serous carcinoma of the ovary is associated with an improved survival. J Ovarian Res 3, 14 (2010).

25. H. Sung et al., Global cancer statistics 2020: GLOBOCAN estimates of incidence and mortality worldwide for 36 cancers in 185 countries. CA Cancer J Clin, (2021).

26. J. M. Taube, Emerging immunologic biomarkers: setting the (TNM-immune) stage. Clin Cancer Res 20, 20232025 (2014).

27. P. Charoentong et al., Pan-cancer Immunogenomic Analyses Reveal Genotype-Immunophenotype Relationships and Predictors of Response to Checkpoint Blockade. Cell Rep 18, 248-262 (2017). 


\section{Acknowledgments}

The collection of the colorectal cancer material and TMA production was supported by U-CAN, through Uppsala Biobank and the Department of Clinical Pathology, Uppsala University Hospital.

\section{Funding}

This study was supported by a postdoctoral grant to A. M. (CAN 2017/1066) and project grants to T. S. (CAN 2018/772) and P. M. (CAN 2018/816) from the Swedish Cancer Society, the Lions Cancer Foundation, Uppsala, Sweden to P. M., the Selanders foundation and P. O. Zetterling Foundation to A. M. U-CAN was supported by the Swedish Government (SRA CancerUU) and locally by Uppsala University and Region Uppsala.

\section{Author contributions}

Conceived study: A.M., T.S.; Designed experiments: A.M., K.L. B.G., P.M., T.S.; Performed experiments: A.M., M.B., S.S.; Image curation: I.H., S.M., S.K.; TMA-cohort construction: P.U.M. (bladder); J.B. (lung), B.N., K.J., F.P. (CRC, melanoma), P.M. (lung)

Patient database curation: J.M., K.H., M.A. (melanoma), P.-H.E., J.H. (endometrial), U.S. (bladder), P.-U.M. (bladder), J.B. (lung), C.H. (gastroesophageal), D.B. (gastroesophageal), J.B. (ovarian), H.S. (ovarian), K.J., F.P.; Data analysis: A.M., A. M.-B., J.E.; Data interpretation: A.M., P.M., T.S.; Wrote the manuscript: A.M., C.L, K.L., B.G., A.P., P.M., T.S. 


\section{Competing interests}

A.M and T.S are co-inventors on a provisional patent application P42105124SE00 "Novel

biomarker" regarding the novel method for the prognosis of survival time of a subject diagnosed with a cancer described herein. No conflicts of interest were disclosed by the other authors.

\section{Data and materials availability}

Data regarding methodology, image analysis, curation and data processing, and raw-data of stroma fraction is available from the corresponding author.

\section{Supplementary Materials}

Materials and methods

Figure S1

Tables S1-S3

References $(4,11,12,14-16,18-24,27)$ 


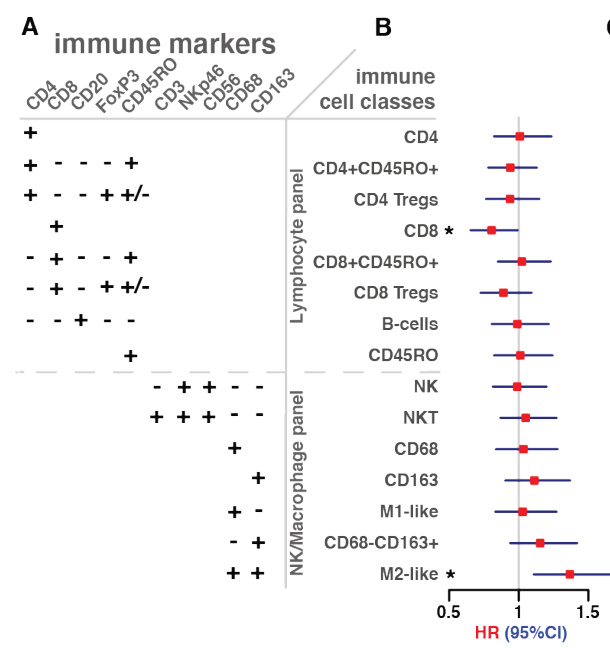

C

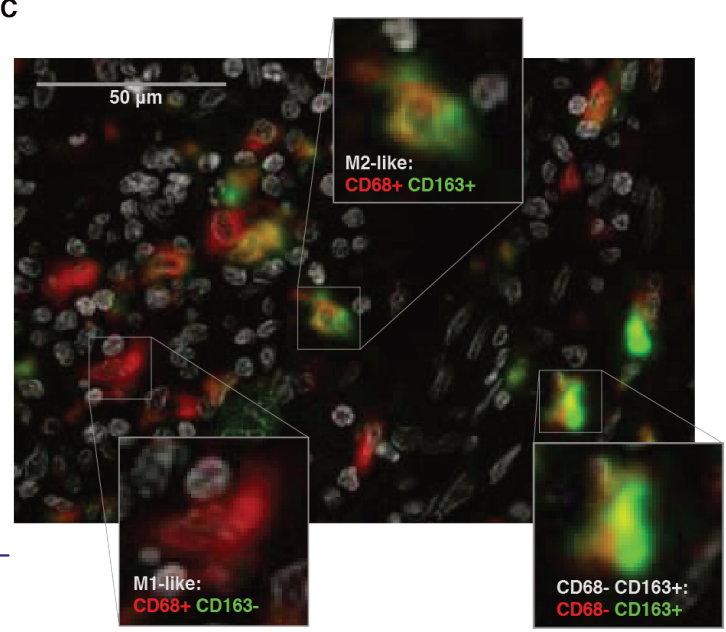

D

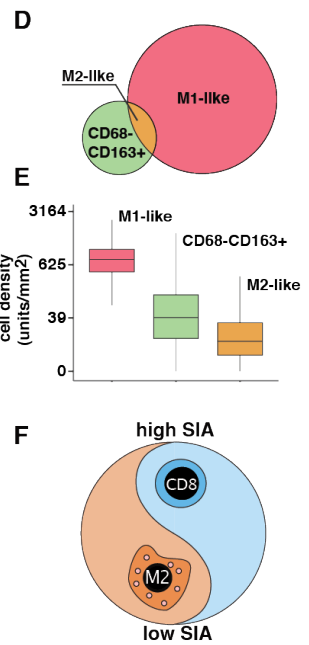

Fig. 1. Prognostic value of CD8+ $\mathrm{T}$ cells and CD68+/CD163+ macrophages revealed by comprehensive characterization of immune cell subsets in 286 therapy-naïve colon cancers.

(A) Immune marker combinations in IHC panels define classes and subclasses of immune cells. (B) Forest plot of univariate associations of tissue immune cell densities translated into three-level categorized values, with OS in patients of stage I-III. Filled squares, hazard ratios (HR); whiskers, 95\% confidence intervals (CI), $* p<0.05$ (Cox regression). (C) Representative multiplex macrophage marker staining of colon cancer tissue. Expression of two markers, CD68 (red) and CD163 (green) with nuclear DAPI staining (white), visualized in pseudocolors, identified three cell types (insets), M1-like macrophages, M2-like macrophages and CD163 ${ }^{+} \mathrm{CD}^{-} 8^{-}$cells. (D) Venn diagram of the counts of cells in the entire cohort expressing CD68 only (red, $n=9.0 \times 10^{5}$ ), CD163 only (green, $n=1.9 \times 10^{5}$ ) or both markers (gold, $n=4.4 \times 10^{4}$ ). (E) Density of three macrophage subsets in patient tumors. Boxes, median and interquartile range (IQR) of the ratios; whiskers, 1.5 IQR. (F) Signature of immune activation (SIA), defined as the ratio of $\mathrm{CD} 8^{+}$cell density to the sum of $\mathrm{CD}^{+}$and M2-like cell densities. 

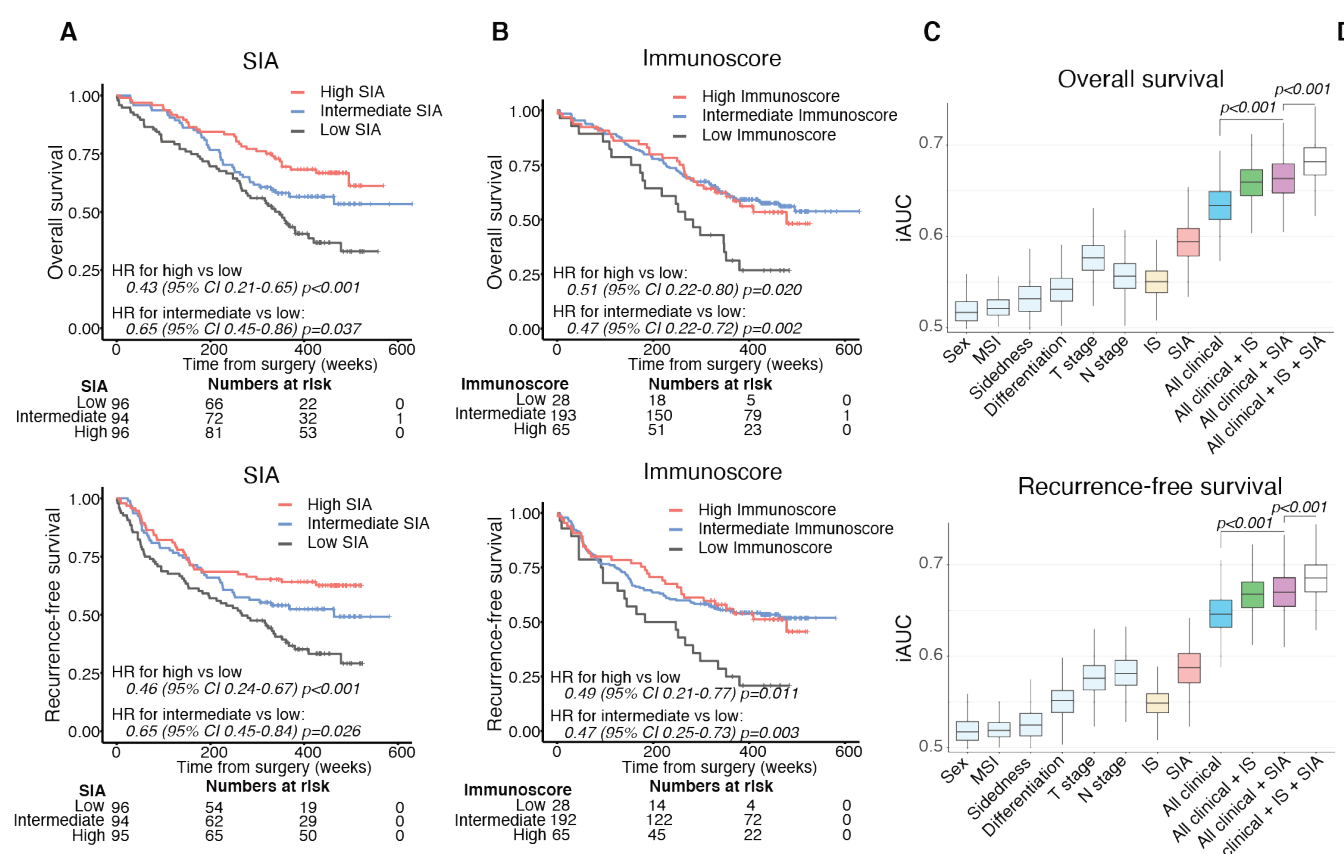

D
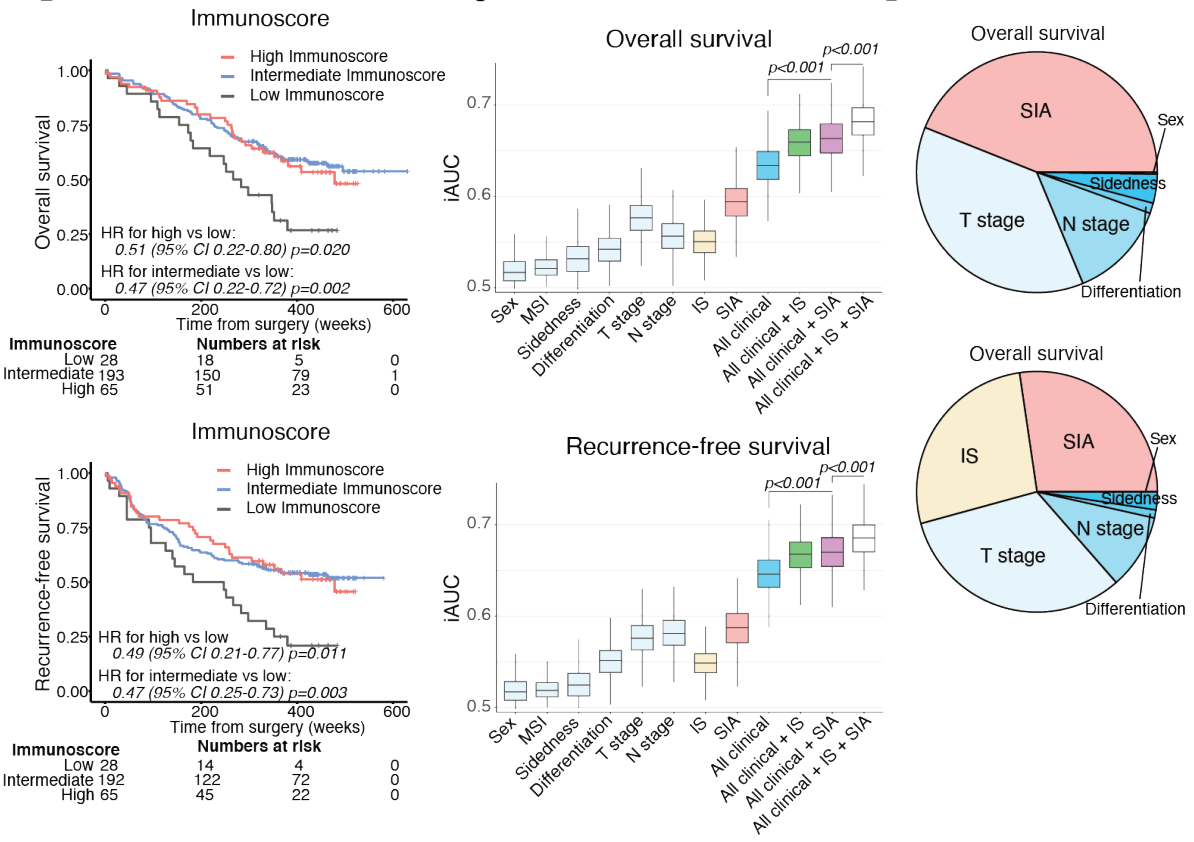

Fig. 2. The SIA is an independent prognostic marker with performance superior to established clinical and immunological predictors for overall (OS) and recurrence-free (RFS) survival in therapy-naïve colon cancer stage I-III patients. (A) OS (upper panel) and RFS (lower panel) for the patients $(n=286)$, stratified into SIA-low, -intermediate and -high groups, with SIA-low used as reference group. (B) OS (upper panel) and RFS (lower panel) for the patients stratified by trichotomized IS. Relative hazards were estimated by Cox proportional hazards model in (A) and (B). (C) Predictive accuracy of SIA, IS and clinical parameters for OS (upper panel) and RFS (lower panel) using iAUC analysis with 1000-fold bootstrap resampling. Univariate Cox proportional hazards models were applied to each of the analyzed factors separately and multivariate models used to evacuate the impact of factor combinations. (D) Relative contribution to the prediction of OS of SIA and clinical parameters (upper) or SIA, IS and clinical parameters (lower) determined using the $\chi^{2}$ proportion test. 

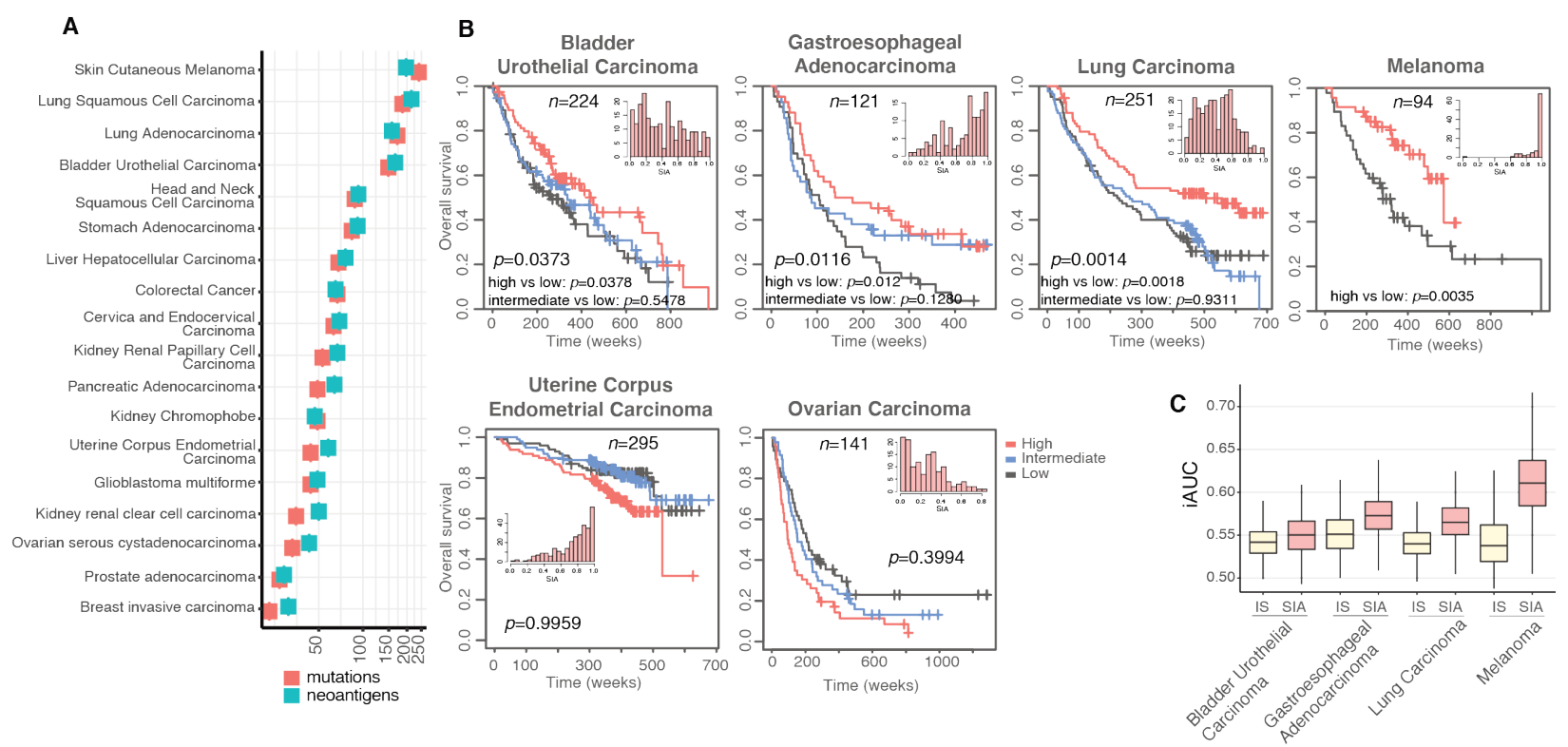

Fig. 3. The SIA is prognostic in bladder cancer, cancer of the gastroesophageal junction, lung

cancer and melanoma. (A) Median numbers of mutations and neoantigens across 19 solid cancers. The data was obtained from The Cancer Immunome Atlas (TCIA) (27). (B) Overall survival stratified by SIA in six tumor types. Tissue microarrays encompassing 91-295 cases of the respective tumor type were stained and the patients in each cohort stratified in terciles according to SIA score, except melanoma, which was stratified in two groups split by the median. Statistical analysis by Cox regression. Insets, histograms of density plots demonstrating the distribution of the continuous SIA metric. (C) Predictive accuracy of IS and SIA for OS in four cancer cohorts, generated using iAUC analysis with 1000-fold bootstrap resampling. 


\section{Table 1. Strong prognostic capacity of SIA in predicting OS and RFS in therapy-naïve stage}

I-III colon cancer patients. Relative hazards, estimated in univariate (for SIA and IS separately) and multivariate (SIA, IS and clinical risk factors) Cox proportional hazards models, using OS and RFS as the endpoints.

\begin{tabular}{|c|c|c|c|c|}
\hline & \multicolumn{2}{|c|}{ Overall survival } & \multicolumn{2}{|c|}{ Recurrence-free survival } \\
\hline Co-variable & $\mathrm{HR}(95 \% \mathrm{CI})$ & $p$ value & $\operatorname{HR}(95 \% \mathrm{CI})$ & $p$ value* \\
\hline \multicolumn{5}{|l|}{ Unadjusted Cox model, SIA } \\
\hline \multicolumn{5}{|l|}{ SIA, three-category } \\
\hline Intermediate vs low & $0.66(0.44-0.98)$ & 0.042 & $0.65(0.44-0.96)$ & 0.03 \\
\hline High vs low & $0.43(0.28-0.67)$ & $<0.001$ & $0.46(0.30-0.70)$ & $<0.001$ \\
\hline \multicolumn{5}{|l|}{ Unadjusted Cox model, IS } \\
\hline \multicolumn{5}{|l|}{ IS, three-category } \\
\hline Intermediate vs low & $0.46(0.28-0.76)$ & 0.002 & $0.49(0.30-0.78)$ & 0.003 \\
\hline High vs low & $0.51(0.29-0.90)$ & 0.02 & $0.49(0.28-0.85)$ & 0.011 \\
\hline \multicolumn{5}{|l|}{ Multivariate Cox model } \\
\hline \multicolumn{5}{|l|}{ SIA, three-category } \\
\hline Intermediate vs low & $0.65(0.42-0.99)$ & 0.047 & $0.65(0.43-0.98)$ & 0.037 \\
\hline High vs low & $0.52(0.33-0.81)$ & 0.004 & $0.55(0.36-0.84)$ & 0.006 \\
\hline \multicolumn{5}{|l|}{ IS, three-category } \\
\hline Intermediate vs low & $0.46(0.27-0.77)$ & 0.003 & $0.49(0.30-0.80)$ & 0.004 \\
\hline High vs low & $0.62(0.33-1.16)$ & 0.137 & $0.62(0.34-1.12)$ & 0.114 \\
\hline \multicolumn{5}{|l|}{ T stage } \\
\hline $\mathrm{T} 2$ vs $\mathrm{T} 1$ & $1.31(0.44-3.95)$ & 0.626 & $1.12(0.37-3.36)$ & 0.84 \\
\hline T3 vs T1 & $1.20(0.56-2.59)$ & 0.636 & $1.25(0.58-2.67)$ & 0.566 \\
\hline T4 vs T1 & $3.51(1.52-8.09)$ & 0.003 & $2.97(1.30-6.79)$ & 0.01 \\
\hline \multicolumn{5}{|l|}{ N stage } \\
\hline $\mathrm{N}+$ vs N0 & $1.74(1.18-2.57)$ & 0.005 & $2.13(1.47-3.08)$ & $<0.001$ \\
\hline \multicolumn{5}{|l|}{ Age } \\
\hline Age $>75$ vs Age $=<75$ years & $3.49(2.40-5.09)$ & $<0.001$ & $2.60(1.82-3.72)$ & $<0.001$ \\
\hline \multicolumn{5}{|l|}{ Sex } \\
\hline Male vs female & $0.85(0.58-1.23)$ & 0.38 & $0.90(0.63-1.28)$ & 0.562 \\
\hline
\end{tabular}


bioRxiv preprint doi: https://doi.org/10.1101/2021.03.24.436814; this version posted March 24, 2021. The copyright holder for this preprint (which was not certified by peer review) is the author/funder. All rights reserved. No reuse allowed without permission.

\begin{tabular}{|r|r|r|r|r|}
\hline MSI status & & & & \\
\hline MMR deficient vs proficient & $0.82(0.50-1.35)$ & 0.444 & $0.95(0.60-1.51)$ & 0.84 \\
\hline MMR missing vs proficient & $1.48(0.59-3.73)$ & 0.406 & $1.18(0.47-2.93)$ & 0.729 \\
\hline
\end{tabular}

MSI-microsatellite instability. MMR-mismatch repair.

*Wald $p$ value. 